\title{
Pion observables with the Minkowski Space Pion Model *
}

\section{J. P. B. C. de Melo ${ }^{\dagger}$}

Laboratório de Física Teórica e Computacional - LFTC

Universidade Cruzeiro do Sul / Universidade Cidade de São Paulo, 01506-000 São Paulo, Brazil

E-mail: joao.mellodcruzeirodosul. edu.br

\section{Rômulo M. Moita}

Instituto Tecnológico de Aeronáutica, DCTA

12.228-900 São José dos Campos, SP, Brazil.

E-mail: rdmoyses@hotmail.com

\section{T. Frederico}

Instituto Tecnológico de Aeronáutica, DCTA

12.228-900 São José dos Campos, SP, Brazil.

E-mail: tobiaseita.br

The pion structure in Minkowski space is described in terms of an analytic model of the Bethe Salpeter amplitude combined with Euclidean Lattice QCD results for the running quark mass. In the present work, a pion model previously proposed, which allows for a Nakanishi integral representation, is studied in order to verify the sensitivity of the pion electromagnetic form factor to small variations of the quark self-energy. In addition, we extend the previous work, providing the Nakanishi integral representation for the invariants associated with a decomposition of the pion Bethe-Salpeter amplitude.

Light Cone 2019 - QCD on the light cone: from hadrons to heavy ions - LC2019

16-20 September 2019

Ecole Polytechnique, Palaiseau, France

*This work was supported in part by CAPES, and Conselho Nacional de Desenvolvimento Científico e Tecnológico (CNPq) under grants 308025/2015-6 (JPBCM), 308486/2015-3 (TF). Fundação de Amparo à Pesquisa do Estado de São Paulo (FAPESP) under the thematic projects 2013/26258-4 and 2017/05660-0, and by regular project 2019/02923-5 (JPBCM). Project INCT-FNA Proc. No. 464898/2014-5.

${ }^{\dagger}$ Speaker. 


\section{Introduction}

In the present work, we extend the previous study performed in [1] to test the sensitivity of the pion observables to the model parameters, and, check the limitations of the model presented with the original parameters. The model is built to fit the quark propagator in the space-like region obtained by Lattice QCD calculations in the Landau gauge (see the reference [1] for details), also the analytical model preserves the Lorentz invariance. The results from Lattice calculations used here, have two degenerate light quarks, $\mathrm{u}$ and $\mathrm{d}$, and, the heavy quark $\mathrm{s}[2,3]$.

The quark model propagator is given by $S_{F}(k)=\imath Z\left(k^{2}\right)\left[\not k-M\left(k^{2}\right)+\imath \varepsilon\right]^{-1}$. Using that the pion is very close to the chiral limit, as a simplification, we not considere the momentum dependence of the quark wave function renormalization factor, i.e, $Z\left(k^{2}\right)=1$. Then, the model for the dressed quark propagator is written as $S_{F}(k)=\imath\left(\not k+M\left(k^{2}\right)\right)\left(k^{2}-M^{2}\left(k^{2}\right)+\imath \varepsilon\right)^{-1}$.

The running quark mass model in the space-like region fits lattice calculations $[1,2,3]$, and it is parametrized by the expression,

$$
M\left(k^{2}\right)=m_{0}-m^{3}\left[k^{2}-\lambda^{2}+i \varepsilon\right]^{-1},
$$

where $m_{0}=0.014 \mathrm{GeV}, m=0.574 \mathrm{GeV}$ and $\lambda=0.846 \mathrm{GeV}$, which we name initial parameter set (IP) [1]. In the chiral limit, where the current quark mass vanishes, the scalar part of the selfenergy gives the invariant associated with the pseudoscalar component of the pion-quark-antiquark vertex. In this way, the present model incorporates effects from quark dressing and dynamical chiral symmetry breaking.

The quark propagator can be written in a factorized form, after solving $m_{i}^{2}=M^{2}\left(m_{i}^{2}\right)$ :

$$
S_{F}(k)=\imath \frac{\left(k^{2}-\lambda^{2}\right)^{2}\left(\not k+m_{0}\right)-\left(k^{2}-\lambda^{2}\right) m^{3}}{\prod_{i=1,3}\left(k^{2}-m_{i}^{2}+\imath \varepsilon\right)},
$$

where for the parameter set given above, only real poles at the positions,

$$
m_{1}=0.371 \mathrm{GeV}, \quad m_{2}=0.644 \mathrm{GeV}, \text { and } m_{3}=0.954 \mathrm{GeV},
$$

are found. The propagator in the form

$$
S_{F}(k)=\iota\left[A\left(k^{2}\right) \not k+B\left(k^{2}\right)\right] .
$$

has for the self-energies:

$$
A\left(k^{2}\right)=\frac{\left(k^{2}-\lambda^{2}\right)^{2}}{\prod_{i=1,3}\left(k^{2}-m_{i}^{2}+\imath \varepsilon\right)}, \quad B\left(k^{2}\right)=\frac{\left(\lambda^{2}-k^{2}\right) m^{3}}{\prod_{i=1,3}\left(k^{2}-m_{i}^{2}+\imath \varepsilon\right)}+A\left(k^{2}\right) m_{0},
$$

We can make for $A\left(k^{2}\right)$, the decomposition below,

$$
\frac{\left(k^{2}-\lambda^{2}\right)^{2}}{\prod_{i=1,3}\left(k^{2}-m_{i}^{2}\right)}=\sum_{i=1}^{3} \frac{D_{i}}{\left(k^{2}-m_{i}^{2}\right)}
$$

and solving for $D^{\prime} s$ with the IP set, one gets:

$$
D_{1}=1.4992, \quad D_{2}=-0.594098 \text { and } D_{3}=-0.0949811 .
$$



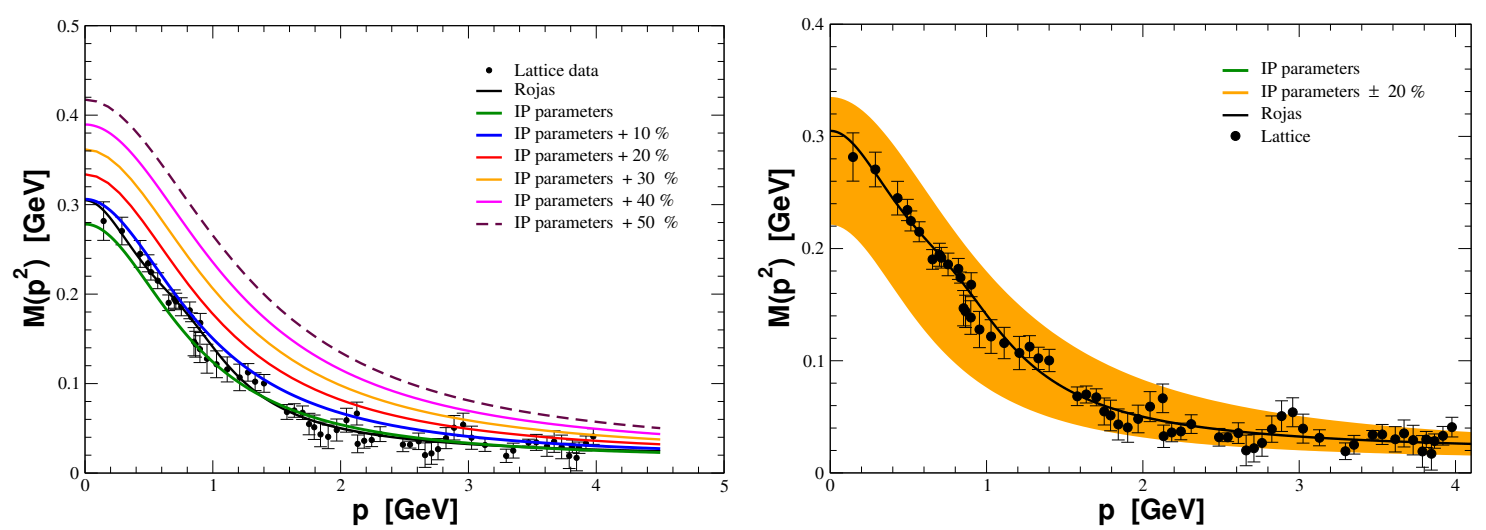

Figure 1: The running quark mass as a function of the momentum $p$, with the parameters from the previous work [1] compared to the results from the parameters variations. Also, in the figure are shown the LQCD results [3] and and the parametrization given in Ref.[2] .

Now, we must decompose $B\left(k^{2}\right)$ from Eq.(1.4) in the same way as above,

$$
\frac{k^{2} m^{3}-\lambda^{2} m^{3}}{\prod_{i=1,3}\left(k^{2}-m_{i}^{2}\right)}+A\left(k^{2}\right) m_{0}=\sum_{i=1}^{3} \frac{D_{i} m_{0}-E_{i}}{\left(k^{2}-m_{i}^{2}\right)}
$$

and we find the following solution for the $E^{\prime} s$ :

$$
E_{1}=0.42401285, \quad E_{2}=-0.331377 \text { and } E_{3}=-0.07863548 .
$$

The spectral decomposition for $A\left(k^{2}\right)$ and $B\left(k^{2}\right)$ reads,

$$
A\left(k^{2}\right)=\int_{0}^{\infty} d \mu^{2} \frac{\rho_{A}\left(\mu^{2}\right)}{k^{2}-\mu^{2}+\imath \varepsilon}, B\left(k^{2}\right)=\int_{0}^{\infty} d \mu^{2} \frac{\rho_{B}\left(\mu^{2}\right)}{k^{2}-\mu^{2}+\imath \varepsilon},
$$

with the spectral densities given by,

$$
\rho_{A}\left(\mu^{2}\right)=-\frac{1}{\pi} \operatorname{Im}\left[A\left(\mu^{2}\right)\right] \text { and } \rho_{B}\left(\mu^{2}\right)=-\frac{1}{\pi} \operatorname{Im}\left[B\left(\mu^{2}\right)\right]
$$

which, in principle, for a non-confining theory should obey the positivity constraints for the KällenLehman (KL) representation, $\mathscr{P}_{a}=\rho_{A}\left(\mu^{2}\right) \geq 0$ and $\mathscr{P}_{b}=\mu \rho_{A}\left(\mu^{2}\right)-\rho_{B}\left(\mu^{2}\right) \geq 0$.

We can write the function $A\left(k^{2}\right)$, as,

$$
\int_{0}^{\infty} d \mu^{2} \frac{\rho_{A}\left(\mu^{2}\right)}{k^{2}-\mu^{2}}=\sum_{i=1}^{3} \frac{D_{i}}{k^{2}-m_{i}^{2}}=\sum_{i=1}^{3} \int_{0}^{\infty} d \mu^{2} \frac{D_{i} \delta\left(\mu^{2}-m_{i}^{2}\right)}{k^{2}-\mu^{2}}
$$

which leads to:

$$
\rho_{A}\left(\mu^{2}\right)=D_{1} \delta\left(\mu^{2}-m_{1}^{2}\right)+D_{2} \delta\left(\mu^{2}-m_{2}^{2}\right)+D_{3} \delta\left(\mu^{2}-m_{3}^{2}\right)
$$

and also

$$
\int_{0}^{\infty} d \mu^{2} \frac{\rho_{B}\left(\mu^{2}\right)}{k^{2}-\mu^{2}}=\sum_{i=1}^{3} \frac{E_{i}}{k^{2}-m_{i}^{2}}=\sum_{i=1}^{3} \int_{0}^{\infty} d \mu^{2} \frac{E_{i} \delta\left(\mu^{2}-m_{i}^{2}\right)}{k^{2}-\mu^{2}}
$$


which has a spectral density given by:

$$
\rho_{B}\left(\mu^{2}\right)=E_{1} \delta\left(\mu^{2}-m_{1}^{2}\right)+E_{2} \delta\left(\mu^{2}-m_{2}^{2}\right)+E_{3} \delta\left(\mu^{2}-m_{3}^{2}\right)+m_{0} \rho_{A}\left(\mu^{2}\right) .
$$

We observe that the parametrization of the quark self energy leads to a violation of the positivity constraints.

The pion Bethe-Salpeter amplitude (BSA) of this model can be written in terms of the Nakanishi integral representation (NIR) [4, 5]. The pion-quark-antiquark vertex denoted by $\Gamma_{\pi}(k, P)$ has the general form below,

$$
\Gamma_{\pi}(k ; P)=\gamma_{5}\left[\iota E_{\pi}(k ; P)+\not P F_{\pi}(k ; P)+k^{\mu} P_{\mu} \not k G_{\pi}(k ; P)+\sigma_{\mu \nu} k^{\mu} P^{v} H_{\pi}(k ; P)\right],
$$

Considering the chiral limit, we have for BSA model the structure below:

$$
\Psi_{\pi}(k ; P)=-\left[A\left(k_{q}^{2}\right) k_{q}+B\left(k_{q}^{2}\right)\right] \frac{\mathscr{N} \gamma_{5} m^{3}}{k^{2}-\lambda^{2}+\imath \varepsilon}\left[A\left(k_{\bar{q}}^{2}\right) k_{\bar{q}}+B\left(k_{\bar{q}}^{2}\right)\right]
$$

where $k_{q}=(k+P / 2), k_{\bar{q}}=(k-P / 2)$ and Eq.(1.3) for the quark propagator. In order to obtain the integral representation of the BSA model, we use Feynman's parameterization, elaborated in the identity below:

$$
\begin{array}{r}
\frac{1}{\left[\left(k+\frac{p}{2}\right)^{2}-\mu^{\prime 2}+\imath \varepsilon\right]\left[k^{2}-\lambda^{2}+\imath \varepsilon\right]\left[\left(k-\frac{p}{2}\right)^{2}-\mu^{2}+\imath \varepsilon\right]}= \\
=\int_{0}^{\infty} d \gamma \int_{-1}^{1} d z \frac{\mathscr{F}\left(\gamma, z ; \mu^{\prime}, \mu, M\right)}{\left[k^{2}+z k \cdot P+\gamma+\imath \varepsilon\right]^{3}},
\end{array}
$$

where

$$
\mathscr{F}\left(\gamma, z ; \mu^{\prime}, \mu\right) \equiv \frac{2 \theta(1+z-2 \alpha) \theta(\alpha-z) \theta(1-\alpha) \theta(\alpha)}{\left|2 \lambda^{2}+M^{2} / 4-\mu^{\prime 2}-\mu^{2}\right|},
$$

and

$$
\alpha\left(\gamma, z ; \mu^{\prime}, \mu\right)=\frac{\gamma-z\left(\mu^{2}-\lambda^{2}-M^{2} / 4\right)+\lambda^{2}}{2 \lambda^{2}+M^{2} / 4-\mu^{2}-\mu^{\prime 2}} .
$$

The BSA from Eq.(1.14) can be decomposed in terms of the Dirac operators,

$$
\begin{aligned}
& \Psi_{\pi}(k ; P)=\gamma_{5} \chi_{1}(k, P)+k_{q} \gamma_{5} \chi_{2}(k, P)+\gamma_{5} k_{\bar{q}} \chi_{3}(k, P)+k_{q} \gamma_{5} k_{\bar{q}} \chi_{4}(k, P)= \\
& =-A\left(k_{q}^{2}\right) k_{q} \frac{m^{3} \mathscr{N} \gamma_{5}}{k^{2}-\lambda^{2}+\imath \varepsilon} A\left(k_{\bar{q}}^{2}\right) k_{\bar{q}}-A\left(k_{q}^{2}\right) k_{q} \frac{m^{3} \mathscr{N} \gamma_{5}}{k^{2}-\lambda^{2}+\imath \varepsilon} B\left(k_{\bar{q}}^{2}\right) \\
& -B\left(k_{q}^{2}\right) \frac{m^{3} \mathscr{N} \gamma_{5}}{k^{2}-\lambda^{2}+\imath \varepsilon} A\left(k_{\bar{q}}^{2}\right) k_{\bar{q}}-B\left(k_{q}^{2}\right) \frac{m^{3} \mathscr{N} \gamma_{5}}{k^{2}-\lambda^{2}+\imath \varepsilon} B\left(k_{\bar{q}}^{2}\right),
\end{aligned}
$$

and the invariants $\chi_{i}(k, P)$ can be written with the Nakanishi integral representation (NIR), with weight functions determined analytically as shown in the following.

In order to obtain the invariants, $\chi_{i}(k, P)$, we introduce $A\left(k_{q}^{2}\right), B\left(k_{q}^{2}\right), A\left(k_{\bar{q}}^{2}\right)$ and $B\left(k_{\bar{q}}^{2}\right)$ in Eq.(1.18), that leads to:

$$
\chi_{i}(k, P)=\int_{0}^{\infty} d \mu^{\prime 2} \frac{\rho_{(A, B)}\left(\mu^{\prime 2}\right)}{\left[\left(k+\frac{P}{2}\right)^{2}-\mu^{\prime 2}+\imath \varepsilon\right]} \frac{m^{3}(-\mathscr{N})}{\left[k^{2}-\lambda^{2}+\imath \varepsilon\right]} \int_{0}^{\infty} d \mu^{2} \frac{\rho_{(A, B)}\left(\mu^{2}\right)}{\left[\left(k-\frac{P}{2}\right)^{2}-\mu^{2}+\imath \varepsilon\right]} .
$$


One can write the scalar functions, $\chi_{i}(k, p)$, with NIR as:

$$
\chi_{i}(k, P)=\int_{-\infty}^{+\infty} d \gamma \int_{-1}^{1} d z \frac{G_{i}(\gamma, z ; M)}{\left[k^{2}+z k \cdot P+\gamma+\imath \varepsilon\right]^{3}},
$$

and after replacing the spectral densities, $\rho_{A}$ and $\rho_{B}$, and integrating over the Dirac delta's in the $\chi_{i}$ 's, we obtain the weight functions:

$$
\begin{aligned}
& G_{1}(\gamma, z ; P)=-m^{3} \mathscr{N} \sum_{i, j}\left(E_{i}+m_{0} D_{i}\right)\left(E_{j}+m_{0} D_{j}\right) F_{i j}, G_{2}(\gamma, z ; M)=-m^{3} \mathscr{N} \sum_{i, j} D_{i}\left(E_{j}+m_{0} D_{j}\right) F_{i j} \\
& G_{3}(\gamma, z ; M)=-m^{3} \mathscr{N} \sum_{i=1}^{3} \sum_{j=1}^{3}\left(E_{i}+m_{0} D_{i}\right) D_{j} F_{i j}, G_{4}(\gamma, z ; M)=-m^{3} \mathscr{N} \sum_{i=1}^{3} \sum_{j=1}^{3} D_{i} D_{j} F_{i j},
\end{aligned}
$$

where $1 \leq i, \leq 3 j$ and $F_{i j}$ are lengthy functions computed with the help of Eq. (1.16), which will be presented elsewhere.

The light-front projection of Eq. (1.20) is the basic ingredient to obtain the valence wave function:

$$
\Psi_{i}\left(z, \vec{k}_{\perp}\right)=\frac{P^{+}}{\sqrt{2}} z(1-z) \int_{-\infty}^{+\infty} \frac{d k^{-}}{2 \pi} \chi_{i}(k, P)=\frac{i}{8 \sqrt{2}}\left(1-z^{2}\right) \int_{-\infty}^{\infty} d \gamma \frac{G_{i}(\gamma, z, P)}{\left[-z^{2} \frac{M^{2}}{4}-\left|\vec{k}_{\perp}\right|^{2}-\gamma\right]^{2}},
$$

where $z=-\frac{2 k^{+}}{P^{+}}$and we have choosen $\vec{P}_{\perp}=0$.

In relation to the the previous work [1], we allowed a variation of the model parameters, to check the robustness of the predictions for the the pion electromagnetic form factor allowing some change in the quark mass function as the basic input from LQCD calculations. Such variations gives also an idea of what to expect in terms of theory uncertainties when comparing with the forthcoming data from the TJLAB laboratory (12 GeV upgrade TJLAB) for energies above their first results [6].
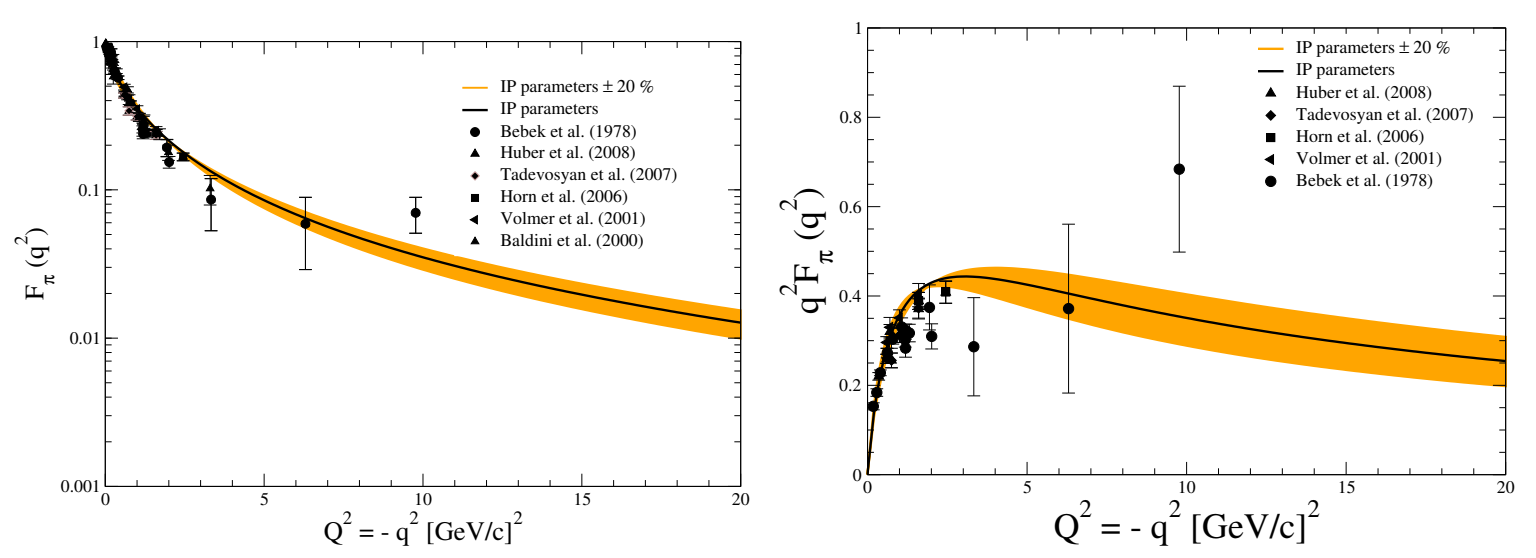

Figure 2: The pion electromagnetic form factor calculated with the model from [1], compared with the experimental data. The band represents results obtained from a $\pm 20 \%$ variation of the parameters.

In the present work, the pion space-like electromagnetic form factor is calculated with a quark electromagnetic current operator that satisfies the Ward-Takahashi identity to ensure current conservation [1]. For the original set of parameters we have a good agreement with the experimental 
electromagnetic radius for the pion, $r_{\pi}^{E x p .}=0.659 \pm 0.004[\mathrm{fm}]$, and also, for the weak pion decay constant, $f_{\pi}^{E x p}=90.276 \pm 0.0707[\mathrm{MeV}](\mathrm{PDG}[11])$. The new results for the pion electromagnetic form factor shown in Fig.(2), electromagnetic radius and the weak decay constant, are found to be consistent with the experimental data $[7,8,9,10,11]$, taking into account a $20 \%$ variation for the model parameters. The resulting band encodes the present experimental data and provides an estimation of the expected error in the prediction of the form factor for large momentum transfers. Also, in the present work, we sketch the derivation of the Nakanishi weight functions of the model, which will allow to investigate the effect of the quark self-energy in these functions preparing the basis for more refined approaches.

\section{References}

[1] Clayton S. Mello, J.P.B.C. de Melo, T. Frederico, Minkowski space pion model inspired by lattice QCD running quark mass, Phys. Lett. B 766 (2017) 86.

[2] E. Rojas, J. P. B. C. de Melo, B. El-Bennich, O. Oliveira and T. Frederico, On the Quark-Gluon Vertex and Quark-Ghost Kernel: combining Lattice Simulations with Dyson-Schwinger equations, JHEP 1310 (2013) 193, [arXiv:1306.3022 [hep-ph]].

[3] M. B. Parappilly, P. O. Bowman, U. M. Heller, D. B. Leinweber, A. G. Williams and J. B. Zhang, Scaling behavior of quark propagator in full QCD, Phys. Rev. D 73 (2006) 054504.

[4] N. Nakanishi, Partial-Wave Bethe-Salpeter Equation, Phys. Rev. 130 (1963) 1230. ibid., A General survey of the theory of the Bethe-Salpeter equation, Prog. Theor. Phys. Suppl. 43 (1969) 1.

[5] J. Carbonell and V. A. Karmanov, Solving Bethe-Salpeter equation for two fermions in Minkowski space, Eur. Phys. J. A 46 (2010) 387.

[6] Jozef Dudek et al., Physics Opportunities with the 12 GeV Upgrade at Jefferson Lab, Eur. Phys. J. A 48 (2012) 187, [arXiv:1208.1244 [hep-ex]].

[7] J. Volmer et al. [Jefferson Lab F(pi) Collaboration], Measurement of the Charged Pion Electromagnetic Form-Factor, Phys. Rev. Lett. 86 (2001) 1713, [nucl-ex/0010009].

[8] T. Horn et al. [Jefferson Lab F(pi)-2 Collaboration], Determination of the Charged Pion Form Factor at $Q^{2}=1.60$ and 2.45-(GeV/c $)^{2}$, Phys. Rev. Lett. 97, 192001 (2006) 192001, [nucl-ex/0607005].

[9] V. Tadevosyan et al. [Jefferson Lab F(pi) Collaboration], Determination of the pion charge form-factor for $Q^{* * 2}=0.60-\mathrm{GeV}^{2}-1.60-\mathrm{GeV}^{2}$, , Phys. Rev. C 75, 055205 (2007) 055205, [nucl-ex/0607007].

[10] G. M. Huber et al. [Jefferson Lab Collaboration], Charged pion form-factor between $Q^{* * 2}=$ $0.60-G e V * * 2$ and 2.45-GeV**2. II. Determination of, and results for, the pion form-factor, Phys. Rev. C 78, (2008) 045203, [arXiv:0809.3052 [nucl-ex]].

[11] M. Tanabashi et al. (Particle Data Group), Phys. Rev. D98 (2018) 030001 and 2019 update. 